\title{
Author Correction: Analysis of DNA methylation in cancer: location revisited
}

Alexander Koch (D), Sophie C. Joosten, Zheng Feng, Tim C. de Ruijter,

Muriel X. Draht, Veerle Melotte, Kim M. Smits, Jurgen Veeck, James G. Herman,

Leander Van Neste, Wim Van Criekinge, Tim de Meyer and Manon van Engeland

Nature Reviews Clinical Oncology https://doi.org/10.1038/s41571-018-0004-4 (2018)

Published online 17 April 2018

The originally published article contained an error in the acknowledgements section in which the Universiteitsfonds Limburg/SWOL is incorrectly presented as awarding an SU2CDCS International Translational Cancer Research Dream Team Grant (Stand Up To Cancer (SU2C)-AACR- DT1415, MEDOCC). This has been corrected in the HTML and PDF versions of the manuscript to reflect that Universiteitsfonds Limburg/SWOL is not the funding body that awarded this grant.

https://doi.org/10.1038/s41571-018-0028-9 I Published online 30 April 2018 